\title{
Análise epidemiológica da hanseníase na região caririense em tempos de pandemia da Covid-19
}

Epidemiological analysis of leprosy in the caririense region in pandemic times of Covid-19

Análisis epidemiológico de la lepra en la región caririense en tiempos de pandemia de Covid-19

Raiza Amanda Gonçalves de Souza ${ }^{1 *}$, Tayná de Sousa Alencar da Silva ${ }^{1}$, Thamires Bezerra Almeida Brito $^{1}$, Gledson Micael da Silva Leite ${ }^{1}$, José Eduardo Pereira Alcântara ${ }^{1}$, Maria Izadora Oliveira Batista ${ }^{1}$, Amanda Salgado Nunes ${ }^{1}$, Edilma Gomes Rocha Cavalcante' ${ }^{1}$ Inês Dolores Teles Figueiredo ${ }^{1}$.

\section{RESUMO}

Objetivo: Analisar o impacto da COVID-19 na epidemiologia da Hanseníase. Métodos: Tratou-se de um estudo epidemiológico de cunho descritivo, na busca de dados secundários na base de dados SINAN, disponibilizados no DATASUS/TABNET e realizado uma análise estatística descritiva simples com os dados encontrados na literatura. Resultados: Encontrou-se números de diagnóstico e notificações menores entre anos de 2017 a 2021, sendo principalmente entre os últimos anos do estudo, 2019, 2020 e 2021, exatos anos onde a pandemia da Covid-19 se instalou e intensificou, entre os municípios estudados, Juazeiro do Norte foi a mais relevante, sendo a população mais afetada, na sua maioria homens na meia idade, da cor parda com baixa escolaridade. As formas multibacilares foram mais frequentes, revelando uma mais intensa transmissibilidade da doença, assim as ações preventivas para hanseníase devem ser direcionadas para esse público. Conclusão: A pandemia da COVID-19 trouxe um grande impacto no sistema de saúde, tanto para as gestões, os profissionais de saúde e para com a qualidade de prestações de serviço para os indivíduos, sendo estes na atenção primária, secundária ou terciária. Com isso, no ano de 2021 os casos notificados foram de 51 casos, 67 casos a menos do que no ano de 2017.

Palavra-chave: COVID-19, Hanseníase, Epidemiologia.

\begin{abstract}
Objective: To analyze the impact of Covid 19 on the epidemiology of leprosy. Methods: This was a descriptive epidemiological study, searching for secondary data in the database SINAN, made available in DATASUS/TABNET and performed a simple descriptive statistical analysis with the data found in the literature. Results: Lower numbers of diagnosis and notifications were found between the years 2017 to 2021, mainly between the last years of the study, 2019, 2020 and 2021, the exact years where the Covid-19 pandemic took hold and intensified, among the cities studied, Juazeiro do Norte was the most relevant, being the most affected population, mostly men in middle age, of mixed race with low education. The multibacillary forms were more frequent, revealing a more intense transmissibility of the disease, so preventive actions for leprosy should be directed to this public. Conclusion: The COVID-19 pandemic has had a major impact on the health system, both for management, health professionals and the quality of service provided to individuals, whether in primary, secondary or tertiary care. As a result, in 2021 the reported cases were 51 cases, 67 cases less than in 2017.
\end{abstract}

Keywords: COVID-19, Leprosy, Epidemiology.

1 Universidade Regional do Cariri, Crato - CE. *E-mail: raiza.amanda@urca.br 


\section{RESUMEN}

Objetivo: Analizar el impacto del COVID-19 en la epidemiología de la lepra. Métodos: Este fue un estudio epidemiológico descriptivo, en la búsqueda de datos secundarios en la base de datos del SINAN, disponible en DATASUS/TABNET, y se realizó un análisis estadístico descriptivo simple con los datos encontrados en la literatura. Resultados: Encontramos menores números de diagnósticos y notificaciones entre 2017 y 2021 , principalmente entre los últimos años del estudio, 2019, 2020 y 2021, años exactos donde se asentó y se intensificó la pandemia de Covid-19, entre los municipios estudiados, Juazeiro do Norte fue la más relevante, siendo la población más afectada, en su mayoría hombres de mediana edad, mestizos y con baja escolaridad. Las formas multibacilares fueron más frecuentes, revelando una transmisibilidad más intensa de la enfermedad, por lo que las acciones preventivas de la lepra deben dirigirse a este público. Conclusión: La pandemia de COVID-19 ha tenido un impacto importante en el sistema de salud, tanto para la gestión, los profesionales de la salud y la calidad del servicio prestado a las personas, ya sea en la atención primaria, secundaria o terciaria. Como resultado, en 2021 los casos notificados fueron 51 casos, 67 casos menos que en 2017.

Palabras clave: COVID-19, Lepra, Epidemiología.

\section{INTRODUÇÃO}

A pandemia do Coronavírus 19 (COVID-19), tem levado a mudança em todos os âmbitos da sociedade. Em especial o impacto nunca antes presenciado nos sistemas de saúde atuais. O momento pandêmico trouxe à tona vulnerabilidades e fragilidades dos sistemas nas suas mais íntimas esferas. As novas recomendações dos órgãos públicos, juntamente com todos os esforços para a criação de novas tecnologias eficazes contra a COVID-19, acabam evidenciado lacunas nos programas antes aplicados, além da mais clara necessidade de intervenções nos determinantes sociais da saúde das pessoas mais vulneráveis, as mais expostas as consequências físicas, mentais, econômicas e sociais da pandemia da COVID-19 (MONTANA M, 2020).

Em meio a todos os entrelaces, a COVID-19 tem levantado inúmeros e preocupantes fatores que têm tirado de foco outros desafios para a saúde pública, agora negligenciados. Em especial as práticas realizadas nas Estratégias de Saúde da Família (ESF), que desempenha um papel fundamental como porta de entrada do Sistema Único de Saúde (SUS), atuando na identificação, encaminhamento e tratamento dos casos leves da COVID-19, coordenadora do cuidado e propagadora da prevenção e promoção da saúde de maneira holística e integral (MEDINA MG, et al., 2020).

As atividades da Atenção Primária foram readaptadas as novas recomendações do ministério da saúde e seu fluxo foi reestabelecido para fornecer os atendimentos com o máximo de eficácia, segurança e conforto. Porém, estudos mostram uma intensa resistência da comunidade em aderir a tais atividades mesmo com a adaptação, por receio do contágio, e desse modo o alcance da ESF tem sido reduzido (RODRIGUES G e LIMA RLB, 2021; SARTI TD, et al.2020).

Este contexto nos leva a refletir sobre as doenças negligenciadas como a hanseníase que antes mesmo da pandemia já era conhecida como uma doença prevalente no Brasil, e a não adesão ao seu tratamento pode gerar consequências como: cegueira, perda de sensibilidades, incapacidades físicas e fatores predisponentes para outros agravos, tal agravo se encontra repleto de misticismo e preconceito além de ser esquecida por muitos, mesmo o Brasil sendo o segundo país no mundo com as maiores taxas de adoecidos por hanseníase, estando abaixo apenas da Índia (SILVA JM, et al., 2021).

Desse modo, motivando-nos a questionar se o momento atual interfere de maneira significativa nos registros e notificações da Hanseníase, e assim no seu manejo. Objetivou-se analisar o perfil epidemiológico da hanseníase em tempos da pandemia de COVID-19, justificando-se pela importância de compreender a interferência da pandemia nas atividades voltadas à identificação, tratamento, cura e redução das incapacidades causadas pela hanseníase, as consequências desta falha são imprevisíveis de médio a longo prazo. Visto os agravos que essa endemia já causa ao longo dos anos. 


\section{MÉTODOS}

Trata-se de um estudo epidemiológico descritivo realizado por meio da busca de dados secundáriosna base de dados Sistema de Informações de Agravos e Notificação (SINAN), disponibilizados pelo Departamento de Informática do Sistema Único de Saúde (DATASUS/TABNET), a coleta dos dados se deu durante o período de outubro e novembro de 2021.

A busca no DATASUS/TABNET foi direcionada a Área Descentralizada de Saúde (ADS) da cidade de Juazeiro do Norte, na região do Cariri, que abrange seis municípios: Juazeiro do Norte; Barbalha; Jardim; Caririaçu; Missão Velha e Granjeiro. Assim como os dados coletados foram dentro do recorte temporal de 2017 a 2021. Salienta-se que essa delimitação no período do estudo foi necessária para realização da comparação entre os anos com e sem a influência da COVID-19.

Para a coleta dos dados, utilizou-se os dados referentes ao perfil das pessoas afetadas pelahanseníase e suas notificações dentro do período estabelecido, 2017 a 2021. Assim foram extraídas diferenciadas as informações concernentes as variáveis de idade, sexo, cor/raça, escolaridade, formas clínicas entre os municípios e entre os anos em estudo.

Os dados coletados foram sintetizados e analisados em planilha do Microsoft Excel/office 2016, organizadas com as variáveis em estudo, para a seguinte análise estatística descritiva simples, com o estudo comparativo da frequência relativa e absoluta em cada variável e anos estudados. Esses resultados são expostos e descritos em gráficos construídos a partir das ferramentas do excel/office 2016.

Como se trata de um banco de domínio público, assim, não houve necessidade de submissão ao Comitê de Ética em Pesquisa.

\section{RESULTADOS e DISCUSSÃO}

No Gráfico 1, observa-se a frequência de notificações de casos novos de hanseníase na ADS de Juazeiro do Norte, identificou-se uma média de mais de 100 casos novos notificados até o ano de 2019, e nos anos subsequentes já ouve uma queda nas notificações constadas no SINAN (69 casos em 2020 e 52 casos em 2021) exatamente sendo estes anos o período de pandemia da COVID-19, em contraste com o ano de 2018 apresenta o maior índice de notificação.

Gráfico 1 - Distribuição anual das notificações de Hanseníase na Região de Saúde de Juazeiro do Norte.

Casos notificados no período de 2017 a 2021

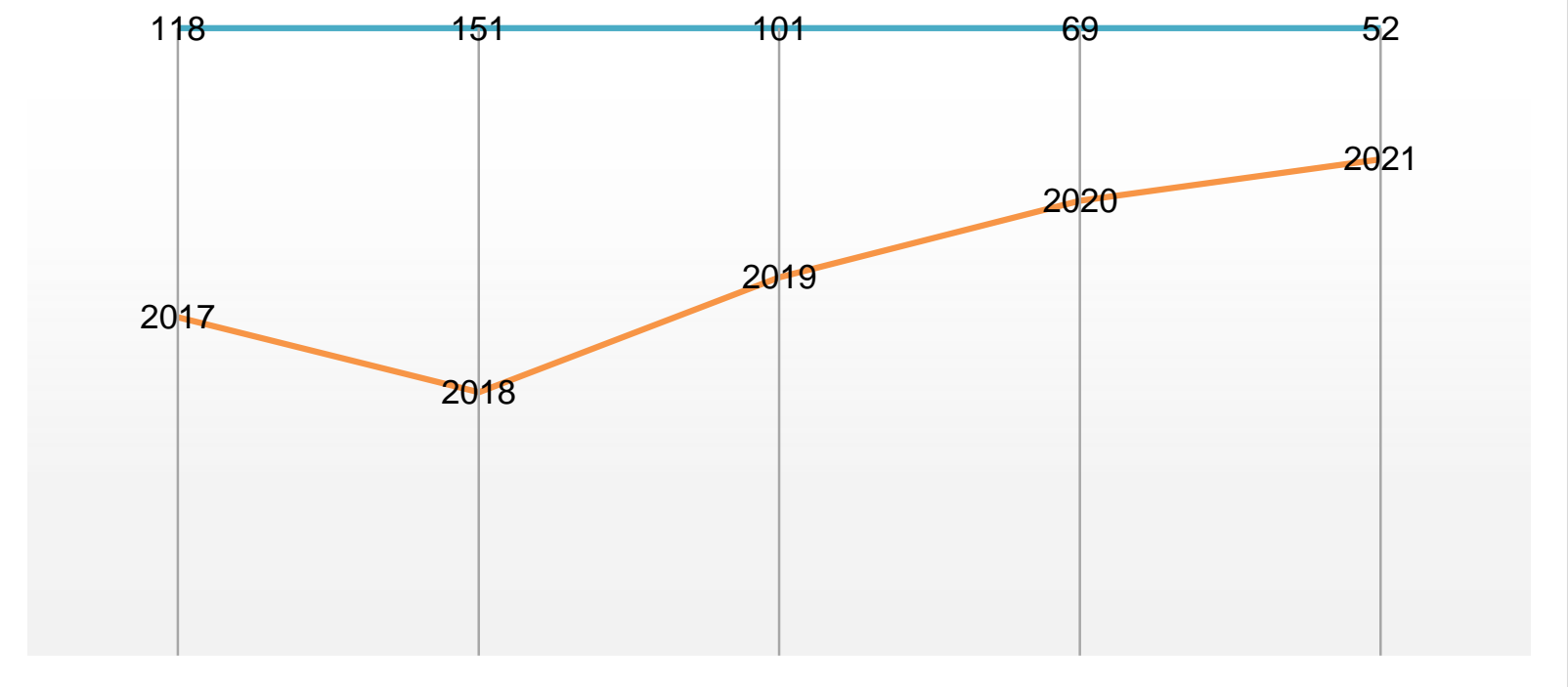

Fonte: Souza RAG, et al., 2022; dados extraídos de Ministério da Saúde/SVS - Sistema de Informação de Agravos de Notificação (Sinan Net), 2021. 
Verifica-se então que no ano de 2018 foram notificados 151 casos e no ano pós início de pandemia, este valor tem uma queda muito brusca, que em 2020 passou a ter somente 69 casos notificados, e no período de 2021 é notório que este valor continua caindo, alcançando 52 casos. No Brasil, em 2019 foram notificados mais de 28 mil casos contra apenas cerca de 14 mil em 2020, dados esses que contribuem com os resultados encontrados no presente artigo, consequentemente conseguimos ver com clareza que a subnotificações de Hanseníase ainda permanece no território brasileiro bem como na ADS de estudo (MINISTÉRIO DA SAÚDE, 2021).

Conforme identifica-se no Gráfico 2, entre as seis cidades que compõem a ADS, Juazeiro do Norte apresentou os maiores resultados em todos os anos, sendo precedido por Missão Velha, Barbalha, Jardim, Caririaçu e Granjeiro. Essa característica pode estar relacionada com a dinâmica territorial dos municípios, sendo Juazeiro do Norte aquele com maior índice populacional.

Gráfico 2 - Distribuição por município das notificações de Hanseníase na Região de Saúde de Juazeiro do Norte.

\section{Casos notificados de 2017 a 2021 da área decentralizada}

\section{Juazeiro do Norte-Ce}

600

500

400

300

200

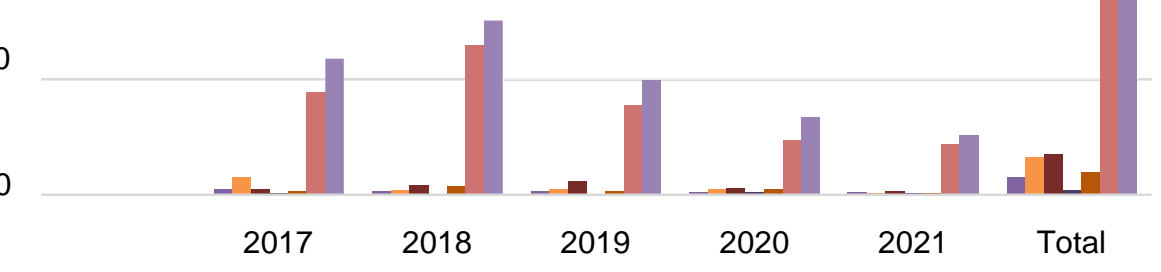

1.... CARIRIACU

BARBALHA

.... MISSAO VELHA

.... GRANJEIRO

.... JARDIM

- .... JUAZEIRO DO NORTE

Total

Fonte: Souza RAG, et al., 2022; dados extraídos de Ministério da Saúde/SVS - Sistema de Informação de Agravos de Notificação (Sinan Net), 2021.

A pandemia da COVID-19 dificultou a realização da busca ativa para novos casos de hanseníase nos municípios, visto as campanhas outrora realizadas envolverem a mobilização comunitária, reunião e momentos de coletividade, assim como inspeção, avaliação e exames físicos para hanseníase, estas práticas foram restringidas pelas novas recomendações para a prevenção da COVID-19. Em contrapartida as práticas de isolamentos social, redução de fluxos humanos e a restrição do fluxo de atendimentos nos serviços de saúde, almejando a redução da transmissibilidade utilizadas medidas de proteção para a não propagação da doença, como o isolamento social e a quarentena, consequentemente pode ter favorecido possíveis subnotificações dessa doença (SILVA JM, et al., 2021).

A Organização Mundial de Saúde recomenda a busca ativa como parte do programa de eliminação da hanseníase e é indescritível que além da identificação desses casos, a busca ativa seja uma ferramenta útil 
para a profilaxia da doença em áreas de alta prevalência ou para o controle de abandonos e de comunicantes porém, é notório que devido a pandemia de covid-19 e as altas taxas de atendimentos relacionados a essa pandemia, dificultaram a atenção a esses outros agravos devido as restrições estabelecidas (MAIA MA, et al., 2020).

Essa dificuldade gerada no atendimento a outros agravos durante a pandemia, foi devido à necessidade de redirecionar o atendimento à COVID-19 visto seu alto índice de pessoas acometidas (13 de maio de 2020 somavam mais de 4 milhões de casos identificados e 283 óbitos até aquele momento), com isso sistema de saúde brasileiro superlotou e ainda procurou manter a qualidade mínima na prestação do serviço, visto também o esgotamento dos profissionais de saúde à sua frente, o que deixou a mercê alguns problemas infeciosos já existentes no país, como é o caso da hanseníase. A percepção da qualidade em saúde nesses casos ocorre de duas perspectivas, a clínica e a populacional, na qual a clínica se preocupa com o impacto da doença e pandemia aos profissionais sobre a saúde dos usuários e a perspectiva populacional que por sua vez, preocupa-se no impacto da saúde sobre a redução das disparidades na saúde e nos subgrupos populacionais, o que interferiu substancialmente no número de notificações relacionadas a hanseníase (MEDONÇA IMS, et al., 2022).

Como citado, a busca ativa juntamente com outras ações tais como a profilaxia através da vacina (BCG) e o aperfeiçoamento de metodologias diagnósticas para identificar aquelas pessoas sob o risco maior de adoecimento, tornou-se fundamental para planejamento de ações integradas e resolutivas para o cuidado das famílias e suas redes sociais acometidas no território de atendimento da APS sendo que tais ações devem ser utilizadas para intensificar a identificação dos casos no período em que os serviços melhor se organizaram ao atendimento de outros agravos infeciosos tais como a hanseníase, que se tornou negligenciada nesse período (SOUZA EA, et al., 2019).

Ressaltando também acerca das formas clínicas identificadas no momento da notificação, visto que as formas clínicas apresentadas estão alinhadas com o potencial de transmissão para contatos, assim como também os níveis de impacto da doença e suas consequentes incapacidades. Desse modo através do Gráfico 3, é possível identificar a frequência das notificações das formas clínicas entre as cidades da ADS em estudo.

Gráfico 3- Distribuição por forma clínica das notificações de Hanseníase na Região de Saúdede Juazeiro do Norte:

Classificação Por Forma Clínica da Hanseníase na Região de Saúde de Juazeiro do Norte (2017-2021)

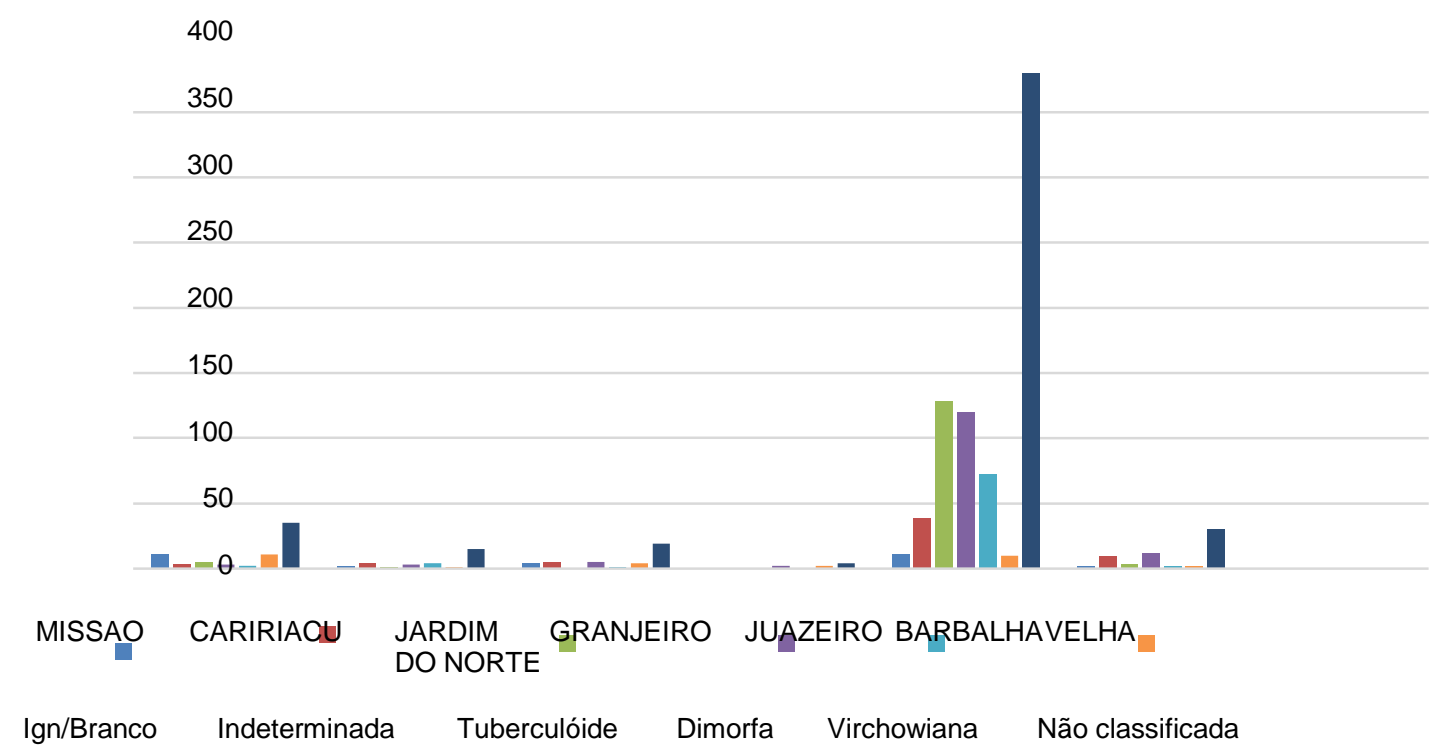

Fonte: Souza RAG, et al., 2022; dados extraídos de Ministério da Saúde/SVS - Sistema de Informação de Agravos de Notificação (Sinan Net), 2021. 
A hanseníase é uma doença de progressão lenta que a medida que progride vai apresentado determinadas manifestações clínicas, sendo elas: indeterminada, tuberculóide, dimorfa e virchowiana, onde cada uma delas são classificadas de acordo com a manifestação sintomática e quanto á carga bacilar entre paubacilar e multibacilar (MINISTÉRIO DA SAÚDE, 2017)., no Gráfico 3, registra-se exatamente os tipos de casos e sua apresentação clínica. Os resultados mostram que a manifestação Dimorfa da hanseníase é a mais prevalente com 145 (30\%) dos registros, seguida pela forma tuberculoide com $137(28,36 \%)$, sequenciadas pela forma Virchowiana com 81 (16,7\%) e a Indeterminada com 60 (12,4\%) dos registros, observa-se também que em 60 (12,4\%) dos registros, essa informação foi ignorada, deixada em branco ou não classificava.

Com relação à análise entre os municípios que compõem a região de saúde em estudo, observou-seque o município de Juazeiro do Norte houve predomínio da forma tuberculoide (128) e dirmorfa (120), cenárioque se repete em Missão Velha tuberculoide (5) e dirmorfa (3) enquanto que nos demais municípios a dimorfa segue como a maior incidência. Vale ressaltar que no município de Granjeiro foram registradas apenas 4 notificações, das quais duas eram sem classificação e as outras duas classificadas como dimorfa, o município de Missão Velha, por sua vez foi o que teve maiores índices de registros com essa informação ignorada, brancaou não classificada, sendo responsável por $33,3 \%$ de todos os registros nessa categoria, o que nos leva a reflexão acerca da qualidade da integralidade das informações contidas nas notificações (MELO MAS, et al., 2018).

As formas de manifestações clínicas da hanseníase seguem a evolução natural da doença quando não identificada e não tratada, desse modo a hanseníase indeterminada é caraterizada pelo aparecimento deaté cinco manchas hipocrômicas com alterações de sensibilidade, contudo não há comprometimento neural.Já a hanseníase tuberculoide é caraterizada por até cinco manchas, porém apresenta o comprometimento neural, essas duas formas são classificadas como paucibacilar, definida pelas poucas manchas e pela presença de pouco ou nenhum bacilo nos exames, em decorrência de sua baixa carga bacilar (GARBELINI GU, et al., 2020).

Já a forma dimorfa é definida pela presença de manchas e placas acima de cinco lesões com importante comprometimento neural, e a hanseníase virchowiana é considerada a forma mais grave e disseminada da doença, com inúmeras manchas e grande comprometimento dos nervos, nariz, rins entre outros órgãos. Essas são as formas mais graves da doença, que são consequência da evolução sem o tratamento adequado, e são classificadas como multibaciliar, aquelas com alto potencial de transmissibilidade (LIMA MHGM, et al., 2020).

Desse modo observa-se que na maioria dos municípios (Juazeiro do Norte, Barbalha, Jardim, Caririaçu e Granjeiro) houve maior incidência das notificações da forma dimorfa, uma das formas multibacilares, com alta transmissibilidade e grave comprometimento neuronal, das formas paucibacilares a forma tuberculoide foi mais incidente entre os municípios, o que revela uma falha no diagnóstico precoce, sendo a sua identificação tardia diretamente relacionada a gravidade da doença e ao tempo de tratamento (MARICATO G, 2020).

Também é possível observar ao longo dos anos (2017-2021) que o perfil das formas de manifestação notificadas foram diferente, pois as formas paucibacilares, com menor índice de transmissão e as formas iniciais da doença, foram mais notificadas nos anos de 2017, 2018, 2019, sendo estes anos responsáveis por $155(78,6 \%)$ dos registros, dado este que nos faz refletir sobre o impacto gerado pela pandemia na identificação precoce da doença bem como a realização do tratamento a fim de se evitar complicações, visto que os anos de 2020 e 2021 houve queda em todos os tipos de notificações, contudo $56(57,1 \%)$ foram nas formas multibacilares, àquelas com maior transmissibilidade, gravidade e impacto na vida do paciente.

Através do Gráfico 4, é possível observar o compilado de informações referentes a incidência da hanseníase quanto a raça/etnia na região de saúde de Juazeiro do Norte, buscando identificar de forma mais clara o perfil dos indivíduos que mais são acometidos pela hanseníase na região de saúde em estudo, sendo este alimentado pelos dados do sistema de notificação que estabelece uma classificação em: Branca, Preta, Amarela, Parda e Indígena, contabilizando também as notificações onde essa informação foi ignorada. 
Gráfico 4 - Distribuição por raça/etnia das notificações de Hanseníase na Região de Saúde de Juazeiro do Norte.

Acompanhamento dos dados de Hanseníase Período: 2017-2021.

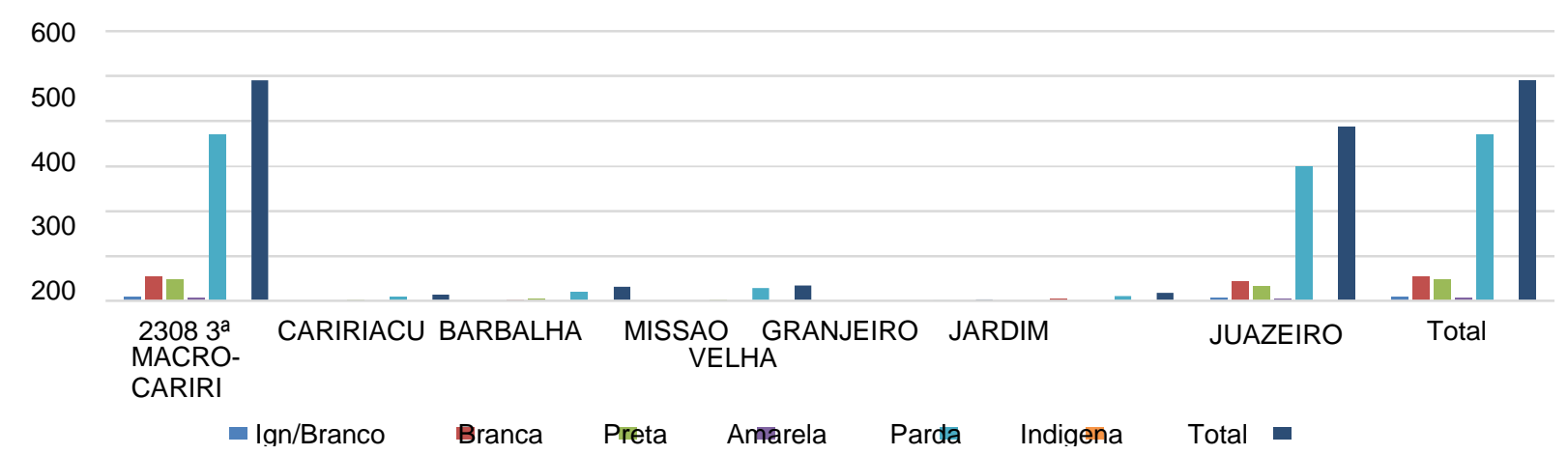

Fonte: Souza RAG, et al., 2022; dados extraídos de Ministério da Saúde/SVS - Sistema de Informação de Agravos de Notificação (Sinan Net), 2021.

No gráfico acima, identifica-se os dados relacionados à raça/etnia, pesquisados no portal do DATASUS. Os mesmos apontaram a diferença de notificações dos casos de hanseníase entre as raças, sendo que a "parda" em primeiro lugar no ano de 2017 à 2021, com 369 casos no total, seguida da "branca" e "preta" com 55 e 49 o número de casos, respectivamente. Essa estimativa, pode ser justificada pelo fato da maioria populacional se auto denominarem da cor parda, como diz Araújo JFF (2019).

Azevedo YP, et al. (2021), em seu estudo sobre o perfil epidemiológico e distribuição espacial da Hanseníase, trouxe dados que corroboraram com o presente trabalho. A raça/etnia mais notificada nos dados avaliados, foi a "parda" tendo como número de casos 609 (66,92\%).

Dentre os indicadores sociais de saúde, destaca-se a escolaridade, visto a sua importância no índice de desenvolvimento humano na qualidade de vida e saúde do indivíduo sob a promoção da sua autonomia, e atuação consciente nas decisões de saúde. Desse modo o Gráfico 5, é possível observar a distribuição da escolaridade dentre os casos notificados de hanseníase na região de saúde em estudo.

Gráfico 5 - Distribuição por escolaridade das notificações de Hanseníase na Região de Saúde de Juazeiro do Norte.

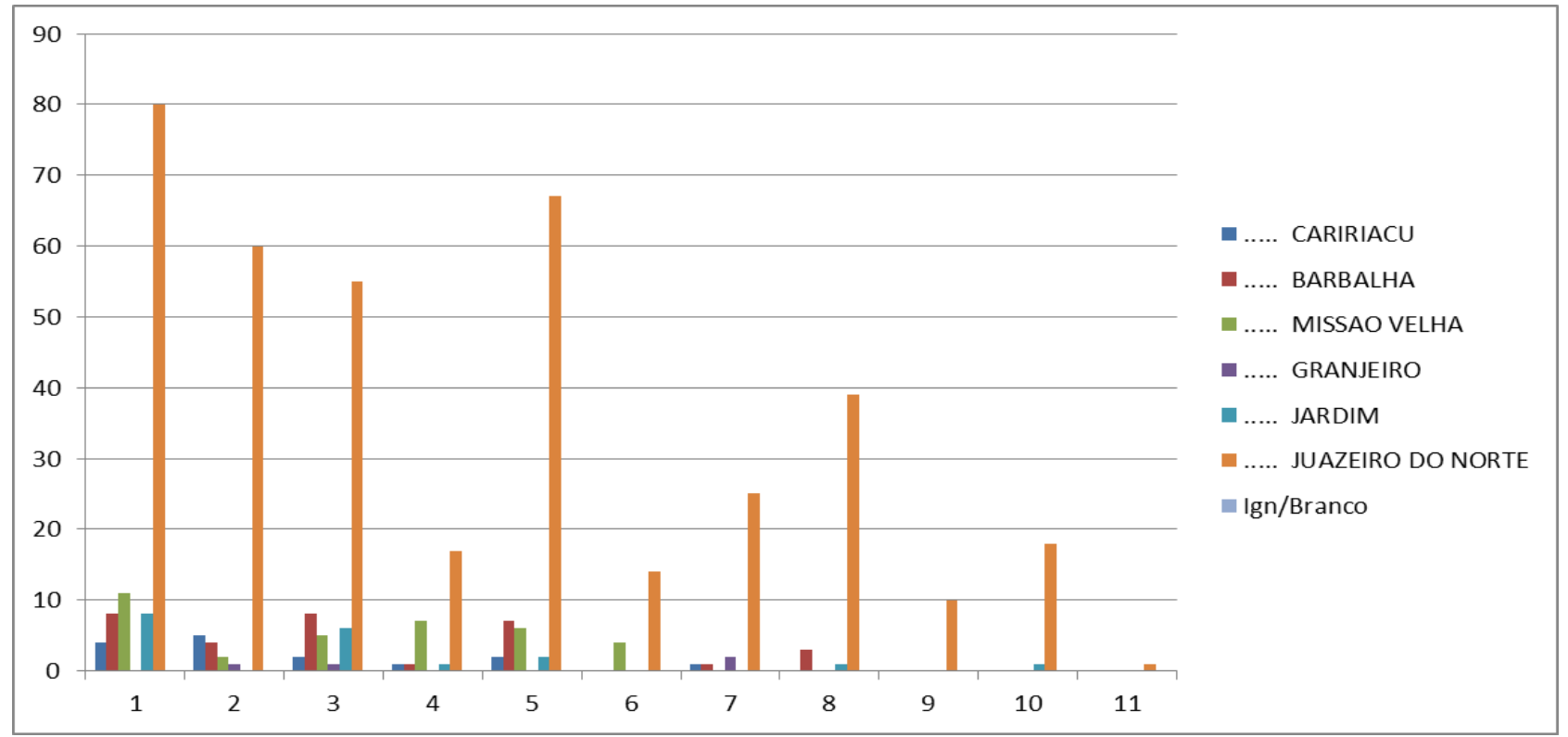

Fonte: Souza RAG, et al., 2022; dados extraídos de Ministério da Saúde/SVS - Sistema de Informação de Agravos de Notificação (Sinan Net), 2021. 
O município de Juazeiro do Norte apresentou a maior taxa de registros ignorados ou em branco. Esse crescente número demonstra uma possível falha dos profissionais durante o preenchimento das fichas de notificação ou recusa dos pacientes em responder sobre a escolaridade. A prevalência de pessoas analfabetas ou com nível de escolaridade baixo apresentados apontam para a estreita relação que existe entre escolaridade e nível socioeconômico a qual afeta diretamente o acesso à saúde. Visto que quanto menor o grau de escolaridade, menores as possibilidades de emprego e menores as condições socioeconômicas (MENGES APN, et al., 2020).

Um fator que é importante ressaltar é que a baixa escolaridade pode contribuir de forma negativa para o surgimento de incapacidades, diminuindo a possibilidade de um diagnóstico precoce. O Ceará apresentou entre 2009 e 2019 uma alta taxa de indivíduos com ensino fundamental incompleto com incapacidades físicas ocasionadas pela hanseníase. No estudo de Lages DS, et al. (2018), foi verificado que o aumento do nível de escolaridade dos indivíduos tende a diminuir a frequência de diagnósticos de hanseníase com incapacidades físicas instaladas. Já pessoas analfabetas e com ensino fundamental possuem uma chance maior de serem diagnosticadas com incapacidades em comparação aquelas com um nível de escolaridade maior.

Compreender a incidência da hanseníase entre os sexos evidencia a influência de fatores relacionados à identidade e estilo de vida condicionando aspectos importantes como fatores potencializadores do risco para a hanseníase assim como para outras patologias como observa-se no estudo de Sales BN, et al. (2020). No que tange a sua influência sob a incidência da hanseníase sob esse aspecto, pode-se associar características individuais de cada género nos hábitos de autocuidado na inspeção da própria pela, toque e avaliação de alterações, assim como o acesso aos serviços de saúde, visto tais considerações o estudo de Melo RLB, et al. (2022) a incidência das notificações de hanseníase é maior no sexo feminino, dado este difere dos encontrados na ADS em estudo de acordo com os dados expresso no Gráfico 6.

Gráfico 6 - Distribuição por sexo das notificações de Hanseníase na Região de Saúde de Juazeiro do Norte.

Sexo

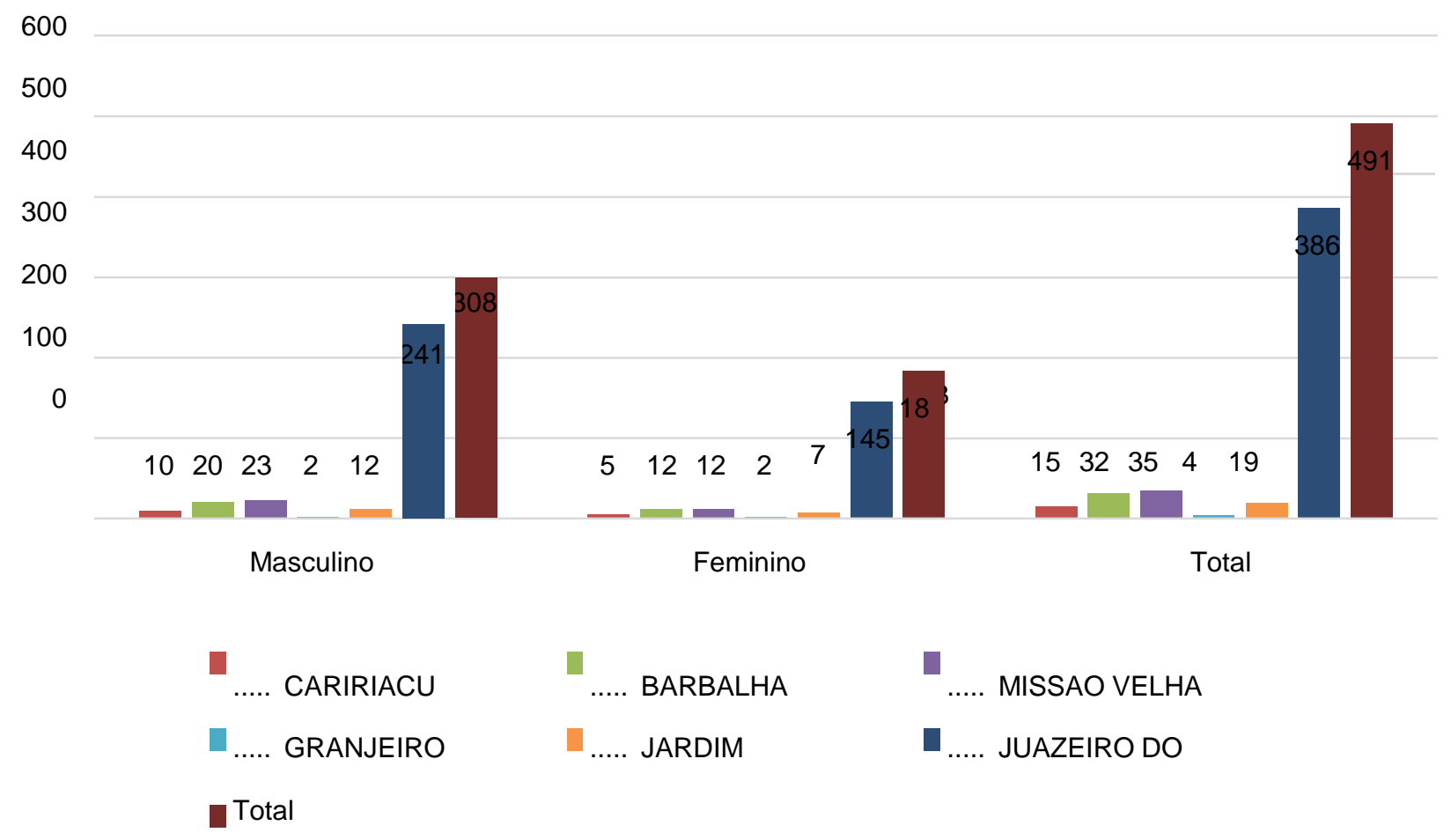

Fonte: Souza RAG, et al., 2022; dados extraídos de Ministério da Saúde/SVS - Sistema de Informação de Agravos de Notificação - Sinan Net, 2021. 
De acordo com a pesquisa realizada acerca dos índices de hanseníase nos sexos masculino e feminino, demonstra-se que há um grande número de casos presentes principalmente no sexo masculino, tendo como somatório o número de 308 pessoas na região de saúde do Juazeiro do Norte, o somatório de casos presentes do sexo feminino, soma-se em 183 casos, presentes dos anos de 2017 a 2021. De acordo ainda com os dados coletados no TABNET, nos anos de 2019 constaram 99 casos, sendo que no ano seguinte, no período de pandemia, os casos registrados reduziram a 67 casos, demonstrando que devido a pandemia e a continuação dos atendimentos, bem como novos atendimentos foram reduzidos, havendo a subnotificação dos casos.

Os dados do Boletim Epidemiológico de Hanseníase (2021), demonstra que entre os anos de 2015 a 2019 no Brasil houve um predomínio de pessoas do sexo masculino, o que corresponde a 53,3\% do total de casos notificados nesse período, percebendo então que os dados coletados acerca da Região de Saúde do Juazeiro do Norte corroboram para os dados gerais do Brasil. Em relação também ao diagnóstico de Hanseníase, em um estudo realizados nos anos de 2015 a 2020 demonstrou-se que a taxa de prevalência do sexo masculino e feminino entre os anos de 2016 e 2019 manteve uma média de $11 \%$ e 7\% respectivamente, em 2020, por sua vez houve uma redução dos números de casos no sexto masculino de $40 \%$ e aumento do sexo feminino, de 43\% (ALMEIDA NCCS, et al., 2021).

\section{CONCLUSÃO}

Desse modo conclui-se que entre os munícipios estudados, Juazeiro do Norte foi onde mais observou-se a discrepância entre os anos de 2017 e 2021, assim também onde a população mais afetada foi a de homens em sua meia idade, seguindo para a terceira idade, e a forma multibacilar foram a mais frequentes, revelando assim que ocorre uma maior transmissibilidade, onde o ser humano do sexo masculino na terceira idade são os principais transmissores da doença, dessarte, as ações preventivas para a hanseníase deve ser direcionadas para este público e com este estudo e toda a sua fundamentação científica e teórica discutida, contribui para que o desenvolvimento de novas estratégias e ferramentas de intervenções são um desafio importante em meio ao cenário pandêmico atual.

\section{REFERÊNCIAS}

1. ALMEIDA NCCS, et al., Análise das taxas de prevalência da hanseníase no pará entre os anos de 2015 e 2020 . Rev. Multi. em Saúde, 2021; 2(3): 72.

2. ARAÚJO JFF. Prevalência de hanseníase nos municípios cearenses: Crato, Juazeiro do Norte e Barbalha entre os anos de 2016 a 2018. Monografia (Graduação em Biomedicina). Centro Universitário Leão Sampaio, Juazeiro do Norte, 2019.

3. AZEVEDO YP, et al. Perfil epidemiológico e distribuição espacial da hanseníase em Paulo Afonso, Bahia. Rev baiana enferm. 2021. 35 (e37805): 1-11.

4. GARBELINI GU, et al. Análise do perfil epidemiológico das formas de apresentação da hanseníase no estado de Goiás. Braz. J. Hea. Rev., Curitiba, 2020; 3(2): 2525-2530.

5. LAGES DS, et al. A baixa escolaridade está associada ao aumento de incapacidades físicas no diagnóstico de hanseníase no Vale do Jequitinhonha. Hu Rev, 2018; 44(3): 303-209.

6. LIMA MHGM, et al. Magnitude e tendência temporal dos indicadores da hanseníase em Goiás: um estudo ecológico do período 2001-2017. Epidemiol. Serv. Saúde, 2020; 29 (5): 1-9.

7. MAIA MA, et al. Extensão universitária: Hanseníase na escola, em busca de um diagnóstico precoce. Revista Brasileira de Extensão Universitária, 2020; 11(1): 25-32.

8. MARICATO G. Entre uma nova epidemia e uma velha endemia: notas sobre as ações dos movimentos de pessoas atingidas pela hanseníase ao longo da pandemia da COVID-19. Cadernos de Campo, 2020; 29(suplemento): 163172.

9. MEDINA MG, et al. Atenção primária à saúde em tempos de covid-19: o que fazer?. Espaço temático: covid-19 contribuições da saúde coletiva. Cad. Saúde Pública 2020; 36(8): 1-5.

10. MELO MAS, et al. Percepção dos profissionais de saúde sobre os fatores associados à subnotificação no Sistema Nacional de Agravos de Notificação. Rev. Adm. Saúde, 2018; 18(71): 1-17.

11. MELO RLB, et al. Distribuição de casos novos da hanseníase em um estado do Nordeste, Research, Society and Development, 2022; 11(1): 1-8. 
12. MENDONÇA IMS, et al. Impacto da pandemia de COVID-19 no atendimento ao paciente com hanseníase: estudo avaliativo sob à ótica do profissional de saúde. Society and Development, 2022; 11(2): 1-10.

13. MENGES APN, et al. Fatores associados à renda e escolaridade em idosos com excesso de peso. Braz. J. of Develop., 2020; 6(9): 65997-66011.

14. MINISTÉRIO DA SAÚDE (MS). Boletim epidemiológico: Hanseníase. 2021. Disponível em: http://www.aids.gov.br/ptbr/pub/2021/boletim-epidemiologico- hanseniase-2021. Acessado em: 10 de jun. de 2021.

15. MINISTÉRIO DA SAÚDE (MS). Guia prático sobre a hanseníase. 2017. Disponível em: https://bvsms.saude.gov.br/bvs/publicacoes/guia_pratico_hanseniase.pdf. Acessado em: 20 de jan. De 2022.

16. MONTANA, M. Vulnerabilidades sociais expostas pelo Covid-19 no Brasil. Campos Neutrais - Revista LatinoAmericana de Relações Internacionais, [S. I.], 2021; 2(1): 91-118.

17. RODRIGUES G, LIMA RLB. Adaptações em uma unidade básica de saúde durante a pandemia de COVID-19: relato de experiência. Health Residencies Journal (HRJ), 2021; 2(10): 140-149.

18. SALES BN, et al. Caracterização epidemiológica da hanseníase nas regiões Norte e Nordeste do Brasil. Research, Society and Development, 2020, 9(8): 1-9.

19. SARTI, TD, et al. Qual o papel da Atenção Primária à Saúde diante da pandemia provocada pela COVID-19? Epidemiologia e Serviços de Saúde [online]. 2020; 29(2): 1-5.

20. SILVA JM dos S, et al. Atenção às pessoas com hanseníase frente a pandemia da COVID-19: uma revisão narrativa. Revista Eletrônica Acervo Saúde, 2021; 13(2): 1-8.

21. SOUZA EA, et al. Desemprenho da vigilância de contatos de casos de hanseníase: uma análise espaço temporal no estado da Bahia, Região Nordeste do Brasil. Cad. Saúde Pública, 2019, 35(9): 1-16. 